\title{
ENFOQUES PARA UNA HISTORIA DE LA FILOSOFÍA CRISTIANA EN AMÉRICA LATINA SS. XVIII A XX
}

\author{
Manuel Domínguez Miranda* \\ doi:10.11144/Javeriana.uph32-65.ehfc
}

\begin{abstract}
RESUMEN
El presente texto corresponde a la Lectio Inauguralis del primer semestre académico de 1988 en nuestra Facultad. Su objetivo es mostrar, desde supuestos teóricos conscientemente asumidos y mediante realizaciones concretas, unas líneas de investigación que pueden hacer inteligible el proceso seguido en Latinoamérica por la filosofía de inspiración explícitamente cristiana. Se busca, ante todo, sacar a la luz lo que está teórica y vitalmente en juego en cada uno de los momentos más significativos de ese proceso. Se destacan también, a modo de ejemplos ilustrativos, algunas figuras filosóficas.

Tras unas breves reflexiones que intentan precisar el significado de los términos "Filosofía Cristiana en Latinoamérica", se expone el desarrollo de esta filosofía desde el S. XVIII hasta el momento presente.

El texto conserva su forma original, sin citas y sin subtítulos. Solo se ha añadido una bibliografía sumaria.

Palabras clave: América latina; filosofía cristiana; historia de la filosofía; escolástica; Cristianismo
\end{abstract}

\footnotetext{
En honor a la memoria de Manuel Domínguez Miranda (1933-2015), Decano y Profesor de la Facultad de Filosofia de la Pontificia Universidad Javeriana; Fundador, Director y Editor de Universitas Philosophica, y Fundador y Director del grupo de investigación Biblioteca Virtual del Pensamiento Filosófico en Colombia (1620-2000), reimprimimos en este número dedicado a las reflexiones actuales de la filosofía en América Latina este artículo, tomado de: Domínguez Miranda, M. (2015). Enfoques para una historia de la Filosofía Cristiana en América Latina SS. XVIII a XX. Universitas Philosophica, 6(10), pp. 21-38.

Para citar este artículo: Domínguez Miranda, M. (2015). Enfoques para una historia de la Filosofía Cristiana en América Latina SS. XVIII a XX. Universitas Philosophica, 32(65), pp. 15-36, ISSN 0120-5323, ISSN en línea: 2346-2426, doi:10.11144/ Javeriana.uph32-65.ehfc
} 


\title{
APPROACHES TO A HISTORY OF CHRISTIAN PHILOSOPHY IN LATIN AMERICA XVIII $^{\mathrm{TH}}$ TO $\mathrm{XX}^{\mathrm{TH}}$ CENTURIES
}

\author{
Manuel Domínguez Miranda
}

\begin{abstract}
RESUMEN
This text corresponds to Lectio Inauguralis of our Faculty in the first academic semester of 1988. Its aim is to show, from theoretical consciously assumed suppositions and through concrete achievements, some lines of research that can make intelligible the process followed in Latin America by the philosophy of explicitly Christian inspiration. It seeks, first of all, to bring to light what is theoretical and vitally at stake in each of the most significant moments of this process. Some philosophical figures are also highlighted as illustrative examples.

After some brief reflections that try to define the meaning of the terms "Christian Philosophy in Latin America", the development of this philosophy is exposed from the 18th century up to the present time.

The text preserves its original form, with no references and subtitles. Only a summary bibliography has been added.

Key words: Latin America; Christian philosophy; history of philosophy; scholastic; Christianity
\end{abstract}


Solo el escuchar la EXPResión "Filosofía Cristiana" suscita en algunos espíritus filosóficos un sentimiento de repulsión o, al menos, un impreciso desasosiego, característicos en quienes se hallan ante el peligro inminente de una mistificación del pensamiento o ante la amenaza de una mutilación de la libertad y la creatividad del espíritu humano.

Añadir cualquier calificativo a la palabra filosofía, sobre todo si ese calificativo connota una posición teórica ya establecida o una tradición cultural, resulta, para algunos pensadores, una restricción inaceptable tanto para la radicalidad u originariedad del pensamiento filosófico como para la ilimitada universalidad de la razón. Recelan, pues, por principio, de la posibilidad de conciliar el Cristianismo y la auténtica filosofía.

No cabe duda de que hay hechos y razones históricas que fundamentan y que pueden, en ocasiones, justificar tales rechazos o recelos. Sin embargo, tampoco puede haber duda de que, en muchos casos, estas reacciones ante la idea de una filosofía cristiana tienen su origen en una falsa concepción tanto de la filosofía como del Cristianismo. Muchos de los que así piensan se hallan inconscientemente atrapados en una idea mítica de la filosofía como saber sin supuestos, como un pensar absoluto que parte de cero y que tiene que crearse sus propias raíces y su propio suelo. Se imaginan también al Cristianismo como un sistema ideológico de formulaciones eternas e inmutables custodiado por una autoridad inflexible.

Pero si alguna lección nos puede aportar la historia del pensamiento occidental es, por una parte, que toda filosofía está ya, desde su origen, calificada y, en cierta medida, delimitada; por otra parte, que el pensamiento cristiano es un proceso incesante en busca de reformulaciones teóricas para lo vivenciado por la fe -tratando de hacerlo pensable y significativo para la vida humana- de acuerdo con el desarrollo histórico en cada época.

La filosofía tuvo su origen dentro de una larga tradición tanto de pensamiento mítico-religioso como de experiencia instrumental del mundo. En esa tradición, en esa masa milenaria de experiencias de la vida y de concepciones del mundo, halla el germen de sus propias ideas y de sus propias actitudes. En filosofía toda novedad surge de una actuación sobre las experiencias humanas anteriores, filosóficas o no filosóficas. Por esto, tanto los creadores y los seguidores de escuelas filosóficas 
como los historiadores de la filosofía, tienen que calificar de algún modo los diferentes resultados del filosofar determinándolos según el nombre de aquello que se adopte como supuesto básico, como realidad primigenia, como dato originario, como método o, simplemente, como tradición o magisterio para el propio filosofar. Así, las distintas filosofías serán materialistas, espiritualistas, empiristas, idealistas, lingüísticas, existencialistas, griegas, cristianas, kantianas, nietzscheanas o con mil calificativos más. De lo que no puede hablarse es simplemente de Filosofía. El filosofar, eterno e irrecusable, produce siempre filosofías determinadas.

Antes de la filosofía está la vida, la de cada cual y la de toda la humanidad histórica, como lo primariamente dado o, en términos orteguianos, como la "realidad radical”. Filosofar es algo que hacemos desde lo que ya somos y vivimos para poder seguir siendo hombres. Pensar al hombre y a su mundo, extendiéndose, remontándose o descendido hasta donde sea necesario abarcar, subir o ahondar para pensarlos sin mutilaciones (en su totalidad) y sin miedos ni tabúes (con toda radicalidad), es lo que, partiendo de un subsuelo cultural que le antecede, se propone - directa u oblicuamente- toda filosofía. Únicamente por las dimensiones de universalidad y radicalidad que pueda aducir en sus resultados, podrá valorarse a una determinada filosofía. No es, pues, razonable rechazar a una filosofía, cualquiera que ella sea, por presentársenos acompañada de un calificativo -que jamás podrá sustituir al trabajo de pensar y que no puede tener otra función que la de orientarnos sobre los supuestos, la tradición o el punto de partida en que se inscribe un acto en concreto de filosofar.

La filosofía cristiana -o, quizá mejor, las filosofías cristianas- son los resultados del esfuerzo teórico que hacen los cristianos al pensar (desde las posibilidades universales de racionalidad, que comparten con todos los hombres) el contenido vital de las creencias de su tradición, la problemática el mundo concreto histórico-social en que se hallan inscritos y la validez de sus creencias para lograr la perfección o plenitud de todo ser humano dentro de la circunstancia en que a cada uno le corresponda vivir. Como toda auténtica filosofía, la filosofía cristiana encierra el propósito de afrontar racionalmente la totalidad de lo que hay -hombres y naturaleza- $y$ abriga el anhelo de servir de fundamento para un proyecto universalmente válido -no necesariamente unívoco u homogéneo- para el perfeccionamiento del vivir humano, histórico, espiritual y material. 
Hablar de filosofía cristiana en Latinoamérica es hablar de un discurso que se ha propuesto como tarea -no siempre asumida explícitamente por todos los que filosofan, pero nunca ausente de sus intenciones- la elucidación, hasta sus últimas raíces y consecuencias, de las relaciones existentes entre vida humana, vida cristiana y vida latinoamericana. "Hombre cristiano", "hombre latinoamericano" y "hombre universal" son los tres conceptos básicos que esta filosofía se ha propuesto, en todo momento y bajo distintas formulaciones, elucidar e integrar.

El hilo de esta triple tarea se viene realizando en este continente, ya desde el S. XVIII, un esfuerzo filosófico de cuño cristiano que ha llegado a constituirse en una de las grandes fuerzas intelectuales configuradoras del perfil socio-cultural de América Latina.

TRATARÉ DE RECOGER - con la brevedad que impone la circunstancia de esta lección- los momentos más destacados de este proceso, que abarca más de dos siglos. No pretendo hacer una historia detallada, con aportes minuciosos de documentos, sino una somera indicación de las líneas maestras de un largo camino.

Como es bien sabido, la conciencia de una identidad latinoamericana, es decir, la conciencia en el habitante de este subcontinente de que posee un ser propio, criollo y mestizo, cuyos intereses no coinciden necesariamente con los intereses de las metrópolis española y portuguesa, comienza a madurar bien entrado el S.XVIII. De esta conciencia aparecen numerosos documentos, correspondientes a distintos sectores de la vida, a lo largo de todo el territorio latinoamericano. Limitándome al campo intelectual, indicaré solo una muestra tomada del Virreinato de la Nueva España y que, en términos generales, ha sido ya suficientemente analizada. Las obras de tres jesuitas: Francisco Javier Clavijero: Historia Antigua de México, de Juan José de Eguiarra y Eguren: Biblioteca mexicana y de Juan Luis Maneiro: Vidas de mexicanos ilustres, junto con los numerosos artículos que José Antonio Alzate y Ramírez publica en su Gaceta Mexicana para refutar las falsas ideas que sobre los americanos se habían formado los europeos, pueden servir para testimoniar una creciente conciencia de americanidad a lo largo del S. XVIII. 
Mucho más viva aún que esta creciente conciencia de americanidad, es la conciencia de la necesidad de una renovación teórica del pensamiento tradicional cristiano mediante la aceptación de la filosofía moderna; es decir, de la filosofía que va desde Descartes a la Ilustración. Este proceso de renovación da pie, en el campo de la filosofía, a una enconada polémica entre los "escolásticos" y los "modernos" que se extiende aproximadamente desde final del primer tercio del S. XVIII a la segunda década del XIX.

Lo que se halla en juego en el fondo de toda esta polémica es la compatibilidad o incompatibilidad del Catolicismo con el pensamiento y con el espíritu de la modernidad. O, en otros términos, la capacidad de la Iglesia Católica para asimilar los enfoques que presentan los filósofos modernos en torno al origen, la constitución y el destino del hombre, de la naturaleza y de la sociedad. La partida la ganan los "eclécticos" y los "modernos" frente a los "rancios", "sistemáticos" o "escolásticos". Pero no la ganan en contra de la fe católica -son caso aislado y extraños los de aquellos modernos a ultranza que rompen con el Catolicismo por considerarlo radicalmente incompatible con la filosofía moderna y con el espíritu de progreso-, sino contra los viejos moldes, tanto institucionales como teóricos, de la vida católica latinoamericana. Son numerosos, y algunos de ellos de gran talla intelectual, los exponentes de este espíritu que combate a la escolástica sin debilitar por ello, en lo más mínimo, la pujanza de su fe cristiana.

Baste citar, entre ello, al mexicano Juan Benito Díaz de Gamarra y Dávalos (1745-1783), hombre de gran altura especulativa, autor de unos Elementos de filosofía moderna, donde trata de conciliar las posiciones fundamentales del pensamiento cristiano con las ideas y métodos de la filosofía moderna; al costarricense, radicado en Guatemala, José Antonio Liendo y Goicoechea, introductor del método empírico y renovador de los planes de estudio en la Universidad de San Carlos de Guatemala; a los jesuitas chilenos desterrados, Juan Ignacio Molina González, Diego Alquízar Herrera y Felipe Gómez Vidaurre, impugnadores del método escolástico en física, seguidores de las teorías físicas modernas y profundos conocedores el pensamiento ilustrado; y, finalmente, a los científicos y polígrafos peruanos Pedro Peralta y Barnuevo, Isidro de Celis y José Eusebio de Llano y Zapata, críticos acervos de la Escolástica e introductores de Newton en la Universidad. 
EL PENSAMIENTO FILOSÓfico CRISTIANo durante el S. XIX está dominado, en su comienzo, por un ligero repunte de la Escolástica colonial -relativamente eclectizada o modernizada - y, en su final, por el despertar del movimiento neoescolástico. Queda en la mitad una larga etapa meramente defensiva y carente de creatividad a los embates del espíritu racionalista-cientista que, desde distintos frentes (sensismo, materialismo, utilitarismo, positivismo y evolucionismo) trata de arrebatar a la Iglesia Católica su tradicional liderazgo cultural en Latinoamérica.

Para un análisis histórico del pensamiento filosófico cristiano latinoamericano durante el S. XIX creo necesario establecer cuatro periodos fundamentales, que presentan cierta homogeneidad interna, aunque exceden, por ambos extremos, los estrictos límites cronológicos del siglo.

El primer periodo se extiende de 1780 a 1814 y abarca la última etapa de la escolástica colonial. El segundo periodo abarca de 1825 a 1840 y corresponde al proceso de liquidación total de la educación y del pensamiento escolásticos. El tercer periodo, que comprende desde 1841 a 1880, corresponde a una etapa apologética (meramente defensiva) del pensamiento cristiano. El cuarto periodo cubre desde 1881 a 1910 y corresponde al inicio del movimiento neoescolástico.

La presencia todavía vigorosa de la escolástica colonial durante el primer periodo se patentiza tanto en la tenaz resistencia que, desde posiciones aristotélicas, tomistas, escotistas o incluso suarecianas, se ofrece en numerosas universidades, colegios o seminarios de las ideas empiristas, sensualistas o materialistas de Condillac, Helvecio, Destutt de Tracy, Holbach, etc., como - de un modo menos sensato- en el rechazo global del método experimental o a todas las posiciones racionalistas o empiristas propugnado por algunos profesores de filosofía o de teología. Esta lucha está comprobada -aunque no se ha realizado el análisis documental de ella- en Venezuela, Colombia, Ecuador, Bolivia, Perú, Uruguay, Chile y Cuba. En México y en América central se puede señalar también este fenómeno y ha sido estudiado con algún detalle.

Pero es quizá en el Río de la Plata, en el Colegio Bonaerense de San Carlos, donde se ha podido mostrar con mayor evidencia la vitalidad del pensamiento escolástico durante la época a que me estoy refiriendo. Con mayor o menor fortuna 
teórica, según los casos, pero con el claro propósito de responder desde la tradición escolástica a las inquietudes y necesidades de su época, trabajó en ese centro de estudios un significativo grupo de profesores de filosofía. Todos ellos tuvieron notable influjo en las primeras décadas de vida independiente de la nación Argentina y fueron los formadores de algunos de los hombres de mayor importancia para la vida del país hasta la mitad del siglo, aunque alejados ya del pensamiento escolástico.

Más allá de esta relativa vitalidad académica de la filosofía escolástica, conviene resaltar la permanencia y la vitalidad en la conciencia colectiva americana de algunas de las tesis o ideas fundamentales de esa filosofía durante las primeras décadas del siglo. Una de estas ideas, cuyo influjo puede documentarse en el contenido y en las formulaciones de no pocos escritos justificativos de la independencia de las colonias españolas, es la tesis sobre la naturaleza y origen popular de la soberanía. Esta idea, común a la tradición española, y argumentada filosóficamente de modo particularmente brillante por la escuela suareciana y jesuítica, había hecho un nido en lo más profundo del alma hispanoamericana. Tan profundas eran sus raíces que, a pesar de haber sido excluida implacablemente de todos los centros de enseñanza por los tres últimos reyes de la dinastía borbónica, esta idea fue retomada, no solo en su contenido sino en la misma formulación y argumentación, por un buen número de primeros documentos justificativos de la independencia de los territorios hispánicos. Entre los forjadores espirituales y materiales de las naciones latinoamericanas pudo haber diferencias profundas en la determinación del concepto "pueblo", pero lo que nunca estuvo en la duda para ellos fue el origen popular de la soberanía. Las ideas de los ilustrados sobre este punto no contenían una novedad absoluta para los hispanoamericanos.

El proceso filosófico en Brasil durante este periodo presenta características muy distintas a las que aparecen en la América Española. La institucionalización del cultivo y de la enseñanza de la filosofía durante el periodo colonial fue considerablemente más débil en el territorio portugués que en el hispánico. Aunque desde finales del S. XVI hubo en Brasil algunos centros de educación superior creados y dirigidos por la iglesia -sobre todo por los jesuitas-, no existieron verdaderas universidades en ese país con anterioridad a 1920. En concordancia con su debilidad institucional, la filosofía escolástica no llegó en Brasil a moldear tan profundamente como en los países de origen español la mentalidad de esas clases 
de dirigentes. Por estas y otras causas, que no es posible explicar aquí, la filosofía escolástica se extingue en Brasil antes de concluir el S. XVIII.

La idea de Que la Independencia de América no es solo un hecho políticoadministrativo sino que implica, además, una nueva visión filosófica de la vida y la adquisición de una nueva mentalidad por parte del hombre americano, es algo que se repite incesantemente a todo lo largo de nuestro subcontinente hasta las postrimerías del S. XIX.

La identificación, en numerosos casos, de la sistemática escolástica con posiciones anti-independentistas o con una mentalidad incompatible con el nuevo espíritu de libertad e igualdad social (abolición de privilegios de la aristocracia y del clero), y con el rechazo a toda posición progresista, lleva en la mayoría de nuevas naciones, desde los primeros años de independencia, a un proceso perfectamente sistematizado de demolición de la Escolástica y de la organización educativa que se inspiraba en ella.

Acabar con la hegemonía educativa y cultural de la Iglesia Católica y abatir los residuos supervivientes de la filosofía escolástica, constituye uno de los programas prioritarios en la mayoría de los nuevos estados durante el segundo y tercer periodos del S. XIX anteriormente enumerados. Una de las muchas expresiones típicas de esta actitud -entre las que se mantienen en un tono relativamente moderado- la hallamos en los labios del colombiano Manuel José Restrepo hacia 1826: "Es doloroso tener que olvidar la mayor parte de lo que aprendimos en la educación colonial... y estudiar de nuevo; pero es necesario para colocarnos a la par de la ilustración del siglo".

Para lograr este objetivo se consideró necesario emprender una intensa campaña educativa con base en filosofías radicalmente laicas y de neto corte naturalista, como las de Destutt de Tracy, Cabanis, Bentham y Mill, o en la de los espiritualistas eclécticos en la línea de Víctor Coussin o Jules Simón, o en los positivismos de Comte o Herbert Spencer.

El pensamiento cristiano de la época -cuando alcanza un nivel teórico que trasciende la defensa meramente jurídica o histórica de la Iglesia institucionalpresenta tres campos básicos de interés: 1) La asimilación de algunos aspectos de 
la filosofía moderna, mostrando su compatibilidad con las tesis básicas del pensamiento cristiano; 2) La necesidad de complementar la filosofía moderna con las ideas fundamentales de la tradición cristiana; y 3 ) La refutación de las filosofías naturalistas, en la medida en que pretenden absolutizar sus puntos de vista como los únicos verdaderamente racionales y los únicos capaces de orientar hacia la felicidad la vida de nuestros pueblos.

Este es, entre otros, el caso de don Andrés Bello y el de don Miguel Antonio Caro, por citar dos ejemplos bien conocidos. En otros casos, como el del obispo de Michoacán, Clemente de Jesús Munguía, se trata de recuperar la doctrina tradicional aristotélico-escolástica en el campo de la filosofía del derecho o de la filosofía social, pero sin llegar a una visión sistemática del conjunto de la problemática filosófica, o, finalmente, en el caso de pensadores de menos talla intelectual que se limitan a proyectar las ideas de la corriente tradicionalista o las concepciones y argumentos de otros apologetas de visión más amplia como Jaime Balmes o Henry Newmann.

Lo que se halla en juego de un modo particularmente dramático durante estos períodos es la identificación cultural del nuevo hombre latinoamericano. Quiere ser moderno y, en la inmensa mayoría de los casos, quiere ser también cristiano. ¿Es posible esa unificación? Y, de ser posible, ¿cuál sería la nueva imagen integral de un hombre americano verdaderamente moderno y verdaderamente cristiano? Las polémicas a las que da pie la búsqueda de respuestas a estas preguntas ocupan la mayor parte de la actividad teórica de los primeros 70 años de la vida latinoamericana independiente.

Lo que nunca parece ponerse en duda en estos años, cualquiera que sea la posición teórica que se adopte, es que las respuestas o modelos tienen que hallarse en pensadores europeos. El hombre americano, cristiano o no cristiano, debe descubrir en Europa su modelo básico de humanidad.

EL MOdELO DE FILOSOfía CRISTIANA que se impone desde las últimas décadas del S. XIX es la neoescolástica, vigorosamente respaldada por el Papa León XIII y por quienes le siguieron en el gobierno universal de la Iglesia Católica. Los esfuerzos apologéticos de los escritores cristianos en el periodo anterior fueron 
evidenciando la necesidad de un pensamiento cristiano totalizante y sistemático que pudiera hacer frente al naturalismo positivista y liberal que dominaba la mentalidad de la época. La vivencia de esta necesidad constituyó el suelo abonado para la recepción de la neoescolástica. La neoescolástica en Iberoamérica no guarda conexión alguna con la escolástica colonial, caducada solo 60 años atrás. La neoescolástica que se desarrolla en estos países es importada directamente de Europa en las últimas décadas del S. XIX, coincidiendo, aproximadamente, con la misma época en que pensadores laicos -en su mayoría respetuosos de nuestra tradición cristiana o ellos mismos positivamente cristianos- inician el proceso de recuperación sistemática de la filosofía europea postmedieval con la introducción en el medio intelectual iberoamericano de algunos esbozos, todavía inseguros, del pensamiento de Kant, Hegel, Leibniz, Descartes, y de otros autores más recientes.

Durante el periodo que estamos reseñando (1880-1910) la presencia efectiva de la neoescolástica en la vida intelectual de Iberoamérica no es muy extensa ni muy profunda. En no pocos casos no pasa de ser un proyecto o un desiderátum. En el campo de las realizaciones concretas son pocos los centros docentes en que se desarrolla y se transmite con profundidad y coherencia esta filosofía que es considerada primordialmente como una "doctrina".

Este doctrinarismo, que sirvió para deslindar con nitidez las posturas intelectuales de los católicos frente a corrientes espiritualistas más o menos imprecisas, se convirtió, en ocasiones, en un obstáculo al querer establecer un diálogo con las filosofías contemporáneas -heredadas de los supuestos, los enfoques y la terminología de la modernidad- cuya introducción en Iberoamérica se iniciaba por esa época tras el creciente abandono del positivismo por parte de la mayoría de los intelectuales.

6.

LA ACTIVIDAD FILOSÓfICA EN ESTE PERIODO, dominado intelectual y socialmente por el cientismo positivista, se había refugiado -dentro de las universidades, escuelas o colegios oficiales de educación superior- en las facultades o escuelas de derecho; por fuera de estas instituciones, en los Seminario y Colegios de la Iglesia. A estos lugares académicos hay que acudir para rastrear los primeros pasos de la filosofía neoescolástica en Iberoamérica. 
Es curiosamente Brasil, y escrita por un pensador laico, donde aparece, con fecha bastante temprana para Iberoamérica, una notable obra filosófica de inspiración y factura claramente tomistas y conscientemente inspirada en algunos de los pioneros de la neoescolástica como Liberatore y Ceferino González. Aludo a las Lições de Filosofía Elementar, Racional e Moral de José Soriano de Souza (1833-1895), publicadas en Recife por la Livraria Académica en 1871. De Souza, que era médico y posteriormente doctor en Filosofía por la Universidad de Lovaina, se desempeñaba como profesor en la Escuela de Derecho de Recife. En su obra plantea con gran nitidez, presentándolo como el tema central la filosofía de su época, el problema de las relaciones entre fe y razón. Sin nombrar explícitamente a sus interlocutores y ponentes -lo que da a la obra un cierto carácter de intemporalidad-, Soriano de Souza polemiza con quienes tratan de establecer un orden mundano fundado sobre una razón plenamente autosuficiente que no requiere de Dios para la organización de la vida humana. Lo más recio de su argumentación va dirigido contra el núcleo central de la tradición racionalista, que él designa con el título de "filosofía cartesio-cousiniana”. Pero el neotomismo de Souza -que, en algunos puntos, está próximo al tradicionalismo- no hizo escuela.

Es en Argentina donde va a tener mayor pujanza y persistencia el pensamiento neoescolástico. Los iniciadores de este movimiento están, de uno y otro modo, ligados a la Universidad de Córdoba, el centro universitario de más tradición en el país.

En Chile debe citarse como indicador del Tomismo al Padre Francisco Ginebra, que durante muchos años desempeñó la cátedra de Filosofía del Colegio de San Ignacio en Santiago. Publicó unos Elementos de Filosofía que, durante largos años, han servido de texto en multitud de colegios católicos en varios países de lengua española.

Como pionero de la docencia del neotomismo en Colombia debe señalarse al presbítero Dr. Joaquín Gómez Otero, quien ya en 1878 exponía en su cátedra del Seminario de Bogotá una metafísica rigurosamente tomista. Pero es sobre todo Monseñor Rafael Ma. Carrasquilla (1857-1930) quien merece ser destacado aquí por su exitoso trabajo como restaurador de la filosofía tomista, en el Colegio Mayor del Rosario, una de las más antiguas y permanentes instituciones universitarias de Colombia, fundada en el S. XVII para propagar el pensamiento de Santo 
Tomás. La personalidad y el esfuerzo cultural integral de Carrasquilla prestigiaron a la filosofía neoescolástica en Colombia y en otros países hispanoamericanos.

Durante el S. XX, la mayor parte del pensamiento filosófico cristiano en Latinoamérica se halla incluido en lo que podemos denominar como movimiento neoescolástico Iberoamericano.

La Neoescolástica es una corriente filosófica que pretende atenerse con fidelidad a las orientaciones emanadas del Vaticano para el cultivo de la filosofía cristiana. Su trabajo se orienta hacia el cumplimiento de tres funciones básicas:

1. Recuperar y transmitir, en la forma más fiel posible, la doctrina, la intención filosófica última y los supuestos teóricos más profundos del pensamiento de Santo Tomás de Aquino y de los otros grandes medievales ortodoxos. Consecuentemente, debe mantener una estrecha conexión con la Teología, pero sin confundirse con ella.

2. Dialogar, desde esta perspectiva escolástica recuperada, con las diferentes corrientes de la tradición filosófica occidental -y particularmente con los autores más representativos del pensamiento contemporáneo-, sobre los problemas que afectan al hombre, a la sociedad y a la cultura en la época actual.

3. Proyectar el pensamiento escolástico, renovado y enriquecido en el diálogo con la tradición y con la filosofía actual (es decir, un pensamiento cristiano actualizado en sus formulaciones, pero enraizado en la tradición medieval), sobre la mentalidad del hombre de hoy, para reconducirlo hacia la trascendencia.

La dinámica cultural generada por la convergencia -no siempre programada- de las actividades filosóficas, de los empeños y desvelos en el trabajo educativo y de los esfuerzos editoriales, hacia el efectivo cumplimiento de esta tripe fundación, es lo que aquí denominamos como movimiento neoescolástico.

Con fin de organizar de un modo suficientemente preciso los datos más importantes sobre el proceso seguido entre nosotros por la filosofía neoescolástica durante el presente siglo, distinguiré en él tres etapas cronológicas fundamentales.

Primera: de 1911 a 1949. Etapa de formación y organización.

Segunda: de 1941 a 1960. Etapa de expansión y diálogo.

Tercera: de 1961 a 1986. Etapa de disolución y concentración. 
No es posible presentar en esta lección el desarrollo seguido por la filosofía neoescolástica durante cada una de estas etapas. Me limitaré a indicar los rasgos más salientes de cada una de ella.

Durante los 40 primeros años del siglo, los neoescolásticos centran sus esfuerzos en la lucha teórica contra el cientifismo positivista y contra el laicismo de ancestro liberal. En la primera de estas batallas coinciden con el esfuerzo de otros muchos filósofos que, como los llamados Fundadores o Patriarcas del pensamiento filosófico contemporáneo en Latinoamérica (el argentino Alejandro Korn, el chileno Enrique Molina, los mexicanos Antonio Caso y José Vaconselos, etc.), tratan, desde diferentes supuestos y puntos de partida, de reivindicar la independencia y la libertad del espíritu. Sin embargo, en la batalla contra el laicismo se enfrentarán con frecuencia a muchos de ellos. Algunos de los espiritualistas laicistas como, por ejemplo, el uruguayo Carlos Vaz Ferreira (1872-1958), el argentino Alejando Korn (1860-1936) o el chileno Enrique Molina (18711956), entre otros, rechazarán las pretensiones de la Neoescolástica cuando esta intente presentarse como la "filosofía perenne". Estimarán, por otra parte, a la Iglesia Católica como a una institución más de nuestra cultura, que vale por la calidad de sus adherentes y por los servicios que pueda prestar a la comunidad y no por algún carácter sobrehumano.

Entre 1920 y 1940 se intensifica notablemente la actividad universitaria de la Iglesia Católica y, paralelamente, la organización y el cultivo de la filosofía neoescolástica. Es el momento en que las grandes órdenes religiosas (Franciscanos, Dominicos, Jesuitas, etc.) abren en muchos países antiguas facultades de filosofía, se crean nuevas universidades católicas y surgen numerosas revistas católicas que abren espacios importantes al pensamiento rigurosamente filosófico, aunque ellas mismas no sean revistas profesionales de filosofía.

Durante esta etapa, la mayor parte de la actividad filosófica en Iberoamérica -en todos los centros educativos, cualquiera que sea su orientación ideológicano se dirige prioritariamente hacia la investigación directa sobre el pensamiento de los grandes filósofos, sino a la formación de profesores de Secundaria con una cierta mentalidad humanística. En los centros universitarios católicos se procura, ante todo, formar profesores para el Bachillerato, pastores de almas y agentes de pastoral imbuidos de una cosmovisión cristiana de cuño neoescolástico. En la práctica, se actúa sobre lo supuesto de que las facultades de filosofía no son crea- 
doras de un pensamiento propio, sino asimiladoras y trasmisoras - con ciertos matices, aplicaciones o actualizaciones locales-del pensamiento producido en las grandes universidades católicas del momento, especialmente en Roma y Lovaina. Sin embargo, los esfuerzos realizados durante estos años hicieron posible el desarrollo de un trabajo técnica y rigurosamente filosófico en las etapas siguientes.

En la década de los cuarenta, el pensamiento neoescolástico había logrado consolidarse en un buen número de países como una de las principales fuerzas impulsadoras del proceso de incorporación de Iberoamérica al movimiento filosófico mundial, la importancia y vitalidad de este proceso fue solemne y reiteradamente reconocida, en la década siguiente, por los más destacados representantes de la filosofía europea en esa época, quienes, en gran número, se hicieron presenten en el Primer Congreso Nacional de Filosofía en Argentina, celebrado en Mendoza entre el 30 de marzo y el 9 de abril de 1949. Varios filósofos de orientación neoescolástica figuraron entre los organizadores del Congreso y un gran número de ellos entre los participantes. Se llega en estos años (1940-1960) a una etapa de esplendor. Durante esta época crecen vertiginosamente, se consolidan o transforman las universidades católicas, los centros y los institutos católicos de educación superior fundados en las décadas anteriores y se crean muchísimos más. Solo en Brasil se cuenta, hacia 1956, 35 facultades católicas de "Filosofía, Ciencias y Letras", que representan el 65\% de las facultades del mismo nombre existentes en ese país.

Es el momento en que, en la mayor parte de América Latina, las disciplinas filosóficas, con el apoyo de una buena infraestructura institucional, llegan a ser cultivadas de un modo riguroso y a ser consideradas, al menos de manera programática, como el núcleo medular de las instituciones universitarias y como estructuradoras del pensamiento humanista que debería inspirar el proceso de desarrollo de los pueblos latinoamericanos. La filosofía neoescolástica es durante este período un interlocutor inteligente y respetado entre las distintas corrientes de pensamiento que aspiran a un papel protagónico en la configuración de una cultura iberoamericana autóctona. Este diálogo obligará a los neoescolásticos a profundizar en el carácter específicamente filosófico de su trabajo y a buscar en la tradición de su propia escuela -más allá de los contenidos doctrinales-, las actitudes espirituales que permitieron a las grandes figuras, como Santo Tomás, San Buenaventura, Escoto o Suárez, pensar a su época desde la fe sin renunciar un solo instante a 
la búsqueda sin fin y sin fronteras que exige del filósofo la inacabable gestación de la humanidad. Esta profundización en el propio ser filosófico y la decisión de asumir en su reflexión todas las dinámicas socio-culturales específicas del momento iberoamericano, tendrá hondas repercusiones en la evolución del movimiento neoescolástico.

Durante la década los sesenta, se producen grandes cambios en la orientación teórica y en la práctica culturas de las universidades católicas, de los seminarios y de los centros de cultura y de investigación dirigidos por la Iglesia. La orientación de los estudios filosóficos queda en manos de una nueva generación de profesores que, durante largos años de estudio en las universidades europeas, han reelaborado, en una dirección diferente a la seguida por los neoescolásticos, su enfoque y su comprensión de lo que puede ser una filosofía de inspiración cristiana. Formados en el contacto directo con las últimas corrientes del pensamiento europeo, como la fenomenología, las filosofías de la existencia, los marxismos y hasta los estructuralismo y las diferentes modalidades de la epistemología y las filosofías de la ciencia, estos profesores destierran definitivamente de sus cátedras los manuales neoescolásticos. Desaparecidas las prevenciones apologéticas frente a las filosofías contemporáneas y, en general, frente a toda tradición filosófica postescolástica, los profesores de esta generación, apoyados en algunas de estas filosofías o en serena actitud crítica frente a ellas, buscarán con ahínco un nuevo lenguaje y un nuevo tipo de discurso -no siempre rigurosa y técnicamente filosófico- que permita, a una fe madura, encarnarse y operativizarse en la vida latinoamericana de su momento histórico. Esta actitud -ligada a numerosas y complejas coyunturas socio-culturales- desatará durante esta etapa un proceso de decantación de las posturas rigurosamente filosóficas frente a las urgencias ideológicodoctrinales de la práctica cultural o pastoral. En algunos centros, como es el caso de esta Facultad, el trabajo filosófico, rehuyendo el hablar superficialmente de cualquier cosa, se repliega hacia una tarea de reflexión y de formación que quiere ser rigurosa y "profesional", corriendo el peligro de ser incomprendido por las instituciones y asfixiado por la falta de recursos. 
Durante los 15 Últimos años, los esfuerzos filosóficos de orientación cristiana presentan en América Latina una notoria pluralidad de direcciones, de esbozos y de proyectos que, en ocasiones, aparecen como enfrentados entre sí. Se dan entre ellos diferencias de fondo respecto a la comprensión de algunos conceptos fundamentales como los de Filosofía, o aspiración a lo universal; Cristianismo, o vigencia de una tradición y una institucionalidad; y Latinoamérica, o aspiración a una identidad cultural. Y hay también diferencias con respecto a la importancia o al papel sistemático que los distintos proyectos atribuyen a cada uno de tales conceptos. Sin embargo, se está de acuerdo en la necesidad de conjugar adecuadamente dichos conceptos.

Precisamente, las innumerables formas en que estas ideas básicas se conjugan o entrecruzan al interior de los distintos proyectos, originan la variada gama de matices que da pie al abigarramiento que presenta, a primera vista, el panorama actual de la filosofía cristiana en Latinoamérica.

Pero, si tomamos como clave para la organización de ese panorama la idea de cultura o de filosofía cristiana que subyace en los diferentes proyectos filosóficos, podremos reducir toda esa compleja pluralidad a tres esquemas fundamentales e ir ubicando, dentro de ellos, los diferentes matices y corrientes de acuerdo con la concepción que acerca de lo latinoamericano y acerca de la filosofía se muestre en cada uno de los proyectos. Estos tres esquemas básicos sin ser radicalmente opuestos o mutuamente excluyentes en todos sus aspectos, presentan sin embargo actitudes y posiciones discordantes en cuestiones de fondo que los hacen profundamente diferentes. Veámoslo muy brevemente.

Para un grupo de pensadores que, como Alberto Caturelli, Stanislaus Ladusans, Agustín Basave Fernández del Valle y Octavio Nicolás Derisi entre otros, constituyen el núcleo central de la Asociación Latinoamericana de Filósofos Católicos -cuyos órgano de expresión es la revista "Filosofar Cristiano" que se edita en Córdoba (Argentina)-, la filosofía cristiana contiene un acervo doctrinal o un depósito de verdades, e incluso de formulaciones teóricas, y una visión sacral de la existencia que son invariables. Es función de esa filosofía recuperar, conservar y explicitar ese contenido básico de acuerdo con las exigencias de cada época. Para ellos, la filosofía cristiana -o la sabiduría secular de Occidente vivificada y 
plenificada por la revelación- constituye la plenitud de la filosofía; cualquier filosofía, en la medida en que se aleja de ese núcleo de verdades y de esa visión sacral del mundo, se degrada y pierde el impulso ascensional hacia la verdad absoluta y universal, meta de toda auténtica filosofía. Piensan también que lo cristiano no es algo accidental en el hombre latinoamericano sino un constitutivo esencial de su propio ser cultural; por eso, la filosofía latinoamericana debería ser siempre una filosofía cristiana, es decir, una filosofía que piense la realidad latinoamericana a la luz de la tradición cultural del Cristianismo, en armonía con las directrices generales del magisterio pontificio. Caben dentro de esta corriente desde continuadores, más o menos rigurosos, del pensamiento escolástico hasta cultivadores de diversas modalidades del espiritualismo interiorista, del personalismo y del existencialismo.

Para un segundo grupo de pensadores (que podrían ejemplarizarse -dentro de una gran diferencia de matices- en Alberto Wagner de Reyna, en Ignacio Ellacuría, en el grupo que respalda a la revista Ánthropos, editada en Venezuela, en gran número colaboradores de revistas filosóficas de inspiración católica y en otros muchos cristiano -eclesiásticos o laicos- que se consideran simplemente profesionales de la filosofía), el Cristianismo es para el que filosofa una inspiración de fondo que mantiene el esfuerzo filosófico permanentemente abierto a la posibilidad de una trascendencia, pero no una estricta norma, positiva o negativa, para su filosofar. El filósofo cristiano tiene que insertarse en la corriente del pensamiento moderno y contemporáneo -que configuran el esquema racional del hombre de hoy- para tratar de resolver los problemas que presenta el desarrollo de las posibilidades de perfeccionamiento humano con que cuentan los individuos, las sociedades y la humanidad actual. Para este fin, el filósofo cristiano se apoyará en las filosofías que le ofrezcan mejores recursos teóricos para esa empresa. Su tarea fundamental debe consistir en la iluminación y orientación teóricas del proceso latinoamericano hacia un horizonte de plenitud humana que esté abierto a lo trascendente. Para este grupo, la filosofía cristiana en latinoamérica la producen los cristianos - conocedores y conscientes de su tradición- al filosofar, desde la altura actual del desarrollo histórico de la filosofía, sobre los problemas del continente latinoamericano.

Hay un tercer grupo de pensadores -con frecuencia incomunicados entre sí- que, como algunos seguidores de la filosofía de la liberación latinoamericana, 
como Juan Carlos Scanonne, como Henrique Claudio de Lima Vaz, y algunos otros, consideran indispensable superar los esquemas teóricos y las categorías conceptuales básicas desarrolladas hasta hoy por el sector dominante del pensamiento occidental. Piensan que estas categorías cosifican radicalmente a toda la realidad e impiden un verdadero encuentro de todos los hombres en la igualdad, el amor y la libertad. Tales categorías contienen símbolos y conceptos ya gastados que tampoco pueden transmitir al hombre de hoy la radical novedad, la universalidad y la trascendencia del mensaje cristiano. Para esta corriente, la filosofía cristiana tiene, como una de sus tareas fundamentales, desarrollar una conceptualización nueva, no viciada de ideologías y asequible a todos los hombres, del mensaje universal y eterno del Cristianismo. Con toda seriedad y con todos los auxilios técnicos que se requieran, pero con osadía, el Cristianismo tiene que lanzarse a una nueva aventura del pensamiento. En esta línea se ensayan distintos caminos. Pero hay, quizá, un camión que va resultado particularmente sugestivo: el trabajo en la conceptualización -que en ningún caso podría partir de cero- de las vivencias y símbolos de la sabiduría popular que posee, en Latinoamérica, un innegable trasfondo cristiano.

8.

Al CONCLUIR ESTE ESBOzo HISTÓRICO -excesivamente largo para los límites de esta lección y excesivamente corto para la amplitud de su objeto- se hace indispensable, por exigencia de un elemental sentido de la objetividad histórica, poner de relieve la extensión y la calidad de los aportes que con los individuos y las instituciones cristianas han contribuido en Latinoamérica al desarrollo, la dignificación y la profesionalización de la filosofía. Aunque con frecuencia los historiadores de la filosofía latinoamericana pasen por alto este hecho, es necesario afirmar que ninguna otra fuerza o institución social ha superado, en su conjunto, lo hecho por la Iglesia Católica en Latinoamérica a favor de la filosofía.

Pero la hora presente no puede ser hora de triunfalismos. En numerosos ámbitos directivos de la vida cristiana se va disipando la conciencia acerca del papel vital que juega la filosofía - una rigurosa filosofía- en el seno del Cristianismo. En este punto, facultades de filosofía que, como la nuestra, tiene viva conciencia del carácter rigurosamente filosófico de su trabajo, pero que se inscriben dentro 
del mundo cristiano institucional, afrontan un gran reto. El reto de hacer patente, comunicable y eficaz - dentro de la pluriforme racionalidad contemporáneala exigencia de una racionalidad universal -transunívoca-inherente al Misterio Cristiano. Racionalidad paradójica que se desborda permanentemente tratando de expresar la incesante novedad de la historia. Solo con base en una racionalidad que es consciente de su propiedad ambigüedad y finitud, resulta posible decir con verdad "nosotros los hombres", nosotros los que tenemos que construir la verdad, una vacilante verdad histórica, mediante la confrontación de razones. Ojalá no seamos inferiores a este reto.

\section{Referencias}

Acerboni, L. (1968). La filosofia contemporánea en Brasil. Milano: Vita e Pensiero. Astonquiza Pizarro, F. (1985). Bio-bibliografia de la filosofia en Chile. Santiago de Chile: Universidad de Chile.

Basaver Fernández del Valle, A. (1957). Filosofía del hombre. México D.F.: F.C.E. Campos, F.A. (1968). Tomismo e Neotomismo no Brasil. Sao Paulo: Grijalbo.

Caturelli, A. (1971). La filosofia en la Argentina actual. Buenos Aires: Sudamericana. Echeverría, J. (1976). Las ideas escolásticas y el inicio de la revolución hispanoamericana, Montalbán, 5, pp 279-338.

Fornet Betancourt, R. (1985). Problemas actuales de la filosofía en Hispanoamérica. Buenos Aires: Fepai.

Furlong, G. (1952). Nacimiento y desarrollo de la filosofia en el Río de la Plata 1526-1810. Buenos Aires: Kraff.

Gómez Martínez, J.L. (1981). Pensamiento iberoamericano: aproximación bibliográfica. Cuadernos salmatinos de filosofía, 8, pp. 287-400.

Ladusans, S. (Comp.) (1976). Rumos da filosofía actual no Brasil. Em autoretratos. Sao Paulo: Ed. Loyola.

Lascaris Comneno, T. (1983). Desarrollo de las ideas filosóficas en Costa Rica. San José: Studium.

Marquínez Argote, G. (Comp.) (1985,1987). La filosofía en Colombia. Bibliografía I, II. Bogotá: USTA. 
Miró Quesada, F. (1974). Despertar y proyecto del filosofar latinoamericano. México D.F.: F.C.E.

Miró Quesada, F. (1981). Proyecto y realización del filosofar latinoamericano. México D.F.: F.C.E.

Navarro, B. (1964). Cultura mexicana moderna en el S. XVIII. México D.F.: U.N.A.M.

Paim, A. (1967). Historia das idéias filosóficas no Brasil. Sao Paulo: Grijalbo.

Roig, A. (1981). Teoria y critica del pensamiento latinoamericano. México D.F.: F.C.E.

Salazar Bondy, A. (1967). Historia de las ideas en el Perú contemporáneo. El proceso del pensamiento filosófico. Lima: Moncloa.

Scannone, J.C. (1987-1988). 1. Teología de la liberación y doctrina social de la Iglesia. 2. Evangelización cultura y Teología. 3. Nuevo punto de partida en la filosofia latinoamericana. Madrid/Buenos Aires: Cristiandad/Guadalupe.

Scannone, J.C. (Ed.) (1984). Sabiduría popular, simbolo y filosofía. Diálogo internacional en torno a la interpretación latinoamericana. Buenos Aires: Guadalupe.

Tatelanning, J. (1978). La Ilustración en la universidad de S. Carlos. Guatemala: Editorial Universitaria.

V.V.A.A. (1981). La filosofía del cristianismo, hoy. I Congreso mundial de Filosofía Cristiana, Córdoba, Argentina.

V.V.A.A. (1979). La filosofía en América. IX Congreso Interamericano de Filosofía, Caracas, Venezuela.

V.V.A.A. (1988). Tendencias actuales de la Filosofia en Colombia. IV Congreso Internacional de Filosofía Latinoamericana. Bogotá, USTA.

V.V.A.A. (1963). Estudios de Historia de la Filosofía en México. México D.F.: U.N.A.M.

Vaz, H. (1986). Escritos de Filosofia. Problemas de Fronteira. Sao Paulo: Ed. Loyola.

Vaz, H. (1968). Ontología e Historia. São Paulo: Duas Cidades.

Wagner de Reyna, A. (1976). Analogía y evocación. Madrid: Gredos.

Wagner de Reyna, A. (1949). La filosofía en Iberoamérica. Lima: Soc. Peruana de Filosofía.

Wagner de Reyna, A. (1982). Pobreza y Cultura. Crisis y Concierto. Lima: Universidad Católica. 